\title{
Una revisión de las variaciones recientes de las plataformas de hielo y su efecto en los glaciares tributarios en el sector norte de la Península Antártica (63ㅇ $\left.-70^{\circ} \mathrm{S}\right)$
}

\author{
A review of recent variations of ice shelves and their effect in \\ tributary glaciers in the north part of the Antarctic Peninsula $\left(63^{\circ} \mathrm{S}-70^{\circ} \mathrm{S}\right)$
}

Eñaut Izagirre ${ }^{1,2}$

\section{Resumen}

En el contexto del aumento promedio de la temperatura superficial del planeta (que fue de $0,6 \pm 0,2^{\circ} \mathrm{C}$ en el siglo XX), y el rápido calentamiento climático del norte de la Península Antártica, que en los últimos 50 años ha incrementado unos $2,5^{\circ} \mathrm{C}$, se resalta que la estabilidad de los glaciares tributarios depende directamente del colapso de las plataformas de hielo, siendo estos los que han sufrido una drástica desintegración en los últimos años. Además, se comparan los datos conocidos de los cambios en la dinámica de los glaciares tributarios con las variaciones recientes de las plataformas, junto con discutir los principales mecanismos que afectan en el colapso de las plataformas y la posterior aceleración y adelgazamiento de los glaciares adyacentes.

\section{Palabras clave:}

Península Antártica, variabilidad climática, "efecto tapón", flujo glaciar.

\footnotetext{
Abstract

In the context of the average increase in the surface temperature of the planet (which was $0.6 \pm 0.2{ }^{\circ} \mathrm{C}$ in the 20th century), and the rapid warming of the North of the Antarctic Peninsula (which in the past 50 years has increased about $2.5^{\circ} \mathrm{C}$ ), emphasis is placed on the direct dependence of the stability of the tributary glaciers depends directly on the collapse of ice shelves, which have suffered a drastic break-up in recent years. In addition, known data of changes in the dynamics of the tributary glaciers is compared with recent variations of the platforms,
}

along with discussing the primary mechanisms that affect in the collapse of platforms and the subsequent acceleration and thinning of adjacent glaciers.

\section{Key words:}

Antarctic Peninsula, climatic variability, "buttress effect", glacier flow.

\section{INTRODUCCIÓN}

Son muchos los estudios que se han centrado en estudiar las variaciones de las plataformas de hielo (ice shelves) en la Antártica, y sobre todo, en la Península Antártica debido a su accesibilidad (Vaughan \& Doake, 1996; Cook et al. 2010; Depoorter et al. 2013).

En años recientes, con el colapso y desintegración de las plataformas de hielo de Príncipe Gustavo y Larsen A en 1995, y gran parte de Wordie en 1992 y Larsen B en 2002 (Glasser et al. 2011; Vaughan \& Doake, 1996; Rignot et al. 2004), las miradas también se han centrado en las consecuencias que estos colapsos han provocado en la dinámica de los glaciares adyacentes. Además, con la mejora de los instrumentos de medición y sensores remotos, las mediciones han revelado que la desintegración de las plataformas lleva al adelgazamiento y aceleración

\footnotetext{
Dirección de Programas Antárticos y Subantárticos.

2 Programa de Magister en Ciencias Antárticas, Universidad de Magallanes, Punta Arenas (Chile). izagirre.enaut@gmail.com $\square$
} 
de los glaciares tributarios (De Angelis \& Skvarca, 2003; Scambos et al. 2004; Rignot et al. 2005; Pritchard \& Vaughan, 2007). Estos, mientras permanecen contenidos por las plataformas, muestran generalmente una dinámica estable, pero una vez que lo que se ha llamado "efecto tapón" desaparece, los glaciares aceleran y adelgazan de forma brusca en períodos de pocos años, hasta volver a restablecer su equilibrio después de un retroceso marcado (Rignot et al. 2004).

Así pues, se ha tratado de compilary sintetizar los trabajos que se han elaborado sobre la conexión entre las plataformas de hielo y los glaciares tributarios del norte de la Península Antártica, en el contexto del calentamiento climático registrado, para entender cual es la respuesta y las diferencias que existen en la dinámica de estos glaciares en respuesta a los cambios en la plataformas de hielo.

\section{ÁREA DE ESTUDIO}

A unos $1.000 \mathrm{~km}$ del Cabo de Hornos, y separado por el agitado Paso Drake, la Península Antártica (PA) se extiende hacia el sur hasta conectarse con el inmenso continente Antártico en la Tierra de Ellsworth (Riffenburgh, 2007).

Para el presente trabajo, el área de estudio corresponde a la muy glaciada parte norte de la $\mathrm{PA}$, extendiéndose desde los $63^{\circ} \mathrm{S}$ hasta los $70^{\circ}$ $\mathrm{S}$ (Fig. 1), y comprende el 1\% (120.000 km²) de todo el continente Antártico, si bien recibe el 10\% de las nevadas (Pritchard \& Vaughan, 2007). En contraste con el extenso Hielo Continental (ice sheet), la parte norte de la Península, que tiene $1.100 \mathrm{~km}$ de longitud y escasos $24 \mathrm{~km}$ en su parte más angosta en el extremo norte, es una larga y angosta cadena montañosa (conocida como Antartandes) salpicada por altiplanos centrales que separan el Mar de Bellingshausen, al oeste, y el Mar de Weddell, al este (Riffenburgh, 2007).

Esta escarpada topografía es drenada por más de 400 glaciares -escarpados, de rápido flujo y fuertemente agrietados- que fluyen como glaciares de desagüe (outlet glaciers), alimentando a las plataformas de hielo o terminando en forma de glaciares afectados por la marea (tidewater glaciers). Con un tercio de su área posando a menos de $200 \mathrm{~m}$ sobre el nivel del mar y con temperaturas del aire que exceden los $0^{\circ} \mathrm{C}$ a nivel del mar en verano, la fusión superficial es un componente importante a tener en cuenta en el balance de masa de estos glaciares y el $80 \%$ de su área está clasificada como zona de percolación (Pritchard \& Vaughan, 2007; Cook et al. 2012).

Geográfica y climáticamente, la citada espina dorsal de la Península, una ininterrumpida cadena montañosa por encima de los 1.400-2.000 metros sobre el nivel del mar, forma una marcada barrera climática. Las regiones del oeste y centro tienen un clima marítimo dominado por el Mar de Bellingshausen, y la costa este tiene un clima continental dominado por el Mar de Weddell. Debido a estas influencias, la costa occidental de la Península es generalmente unos $3-5{ }^{\circ} \mathrm{C}$ más templada que la costa oriental a similar latitud y elevación (Vaughan et al. 2003; Morris \& Vaughan, 2003).

\section{CONTEXTO CLIMÁTICO ACTUAL}

Es inevitable que la Temperatura Promedio Global de la Superficie (Global Mean Surface Temperature) ha incrementado desde finales del siglo XIX. Los datos recabados por el Panel Intergubernamental sobre el Cambio Climático (IPCC 2013), combinando las temperaturas globales de la superficie terrestre y oceánica, muestran un incremento de $0,89^{\circ} \mathrm{C}$ en el periodo $1901-2012$, con gran parte de ese aumento $\left(0,72^{\circ} \mathrm{C}\right)$ en el periodo 1951-2012, el cual es atribuido en buena medida al incremento antropogénico de los gases de efecto invernadero. Más aún, desde las últimas décadas, la temperatura media del planeta ha sido la más alta del último milenio (Mann et al. 1999). Sin embargo, a pesar del fuerte calentamiento en la escala de tiempo multi-decadal, existe una sustancial variabilidad multi-anual en la tasa de calentamiento; con muchos periodos que señalan una tendencia no lineal, incluyendo la interrupción del calentamiento desde 1998, que con una tasa de calentamiento de $0,05{ }^{\circ} \mathrm{C}$ por década (en el periodo 1998-2012), es menor que la tendencia desde 1951 (0,12 ${ }^{\circ} \mathrm{C}$ por década).

Además, dentro de la tendencia general mencionada, se han observado tres áreas regionales o sub-continentales en latitudes altas con rápido calentamiento atmosférico. Por un lado, el noroeste de Norte América y la zona central del 


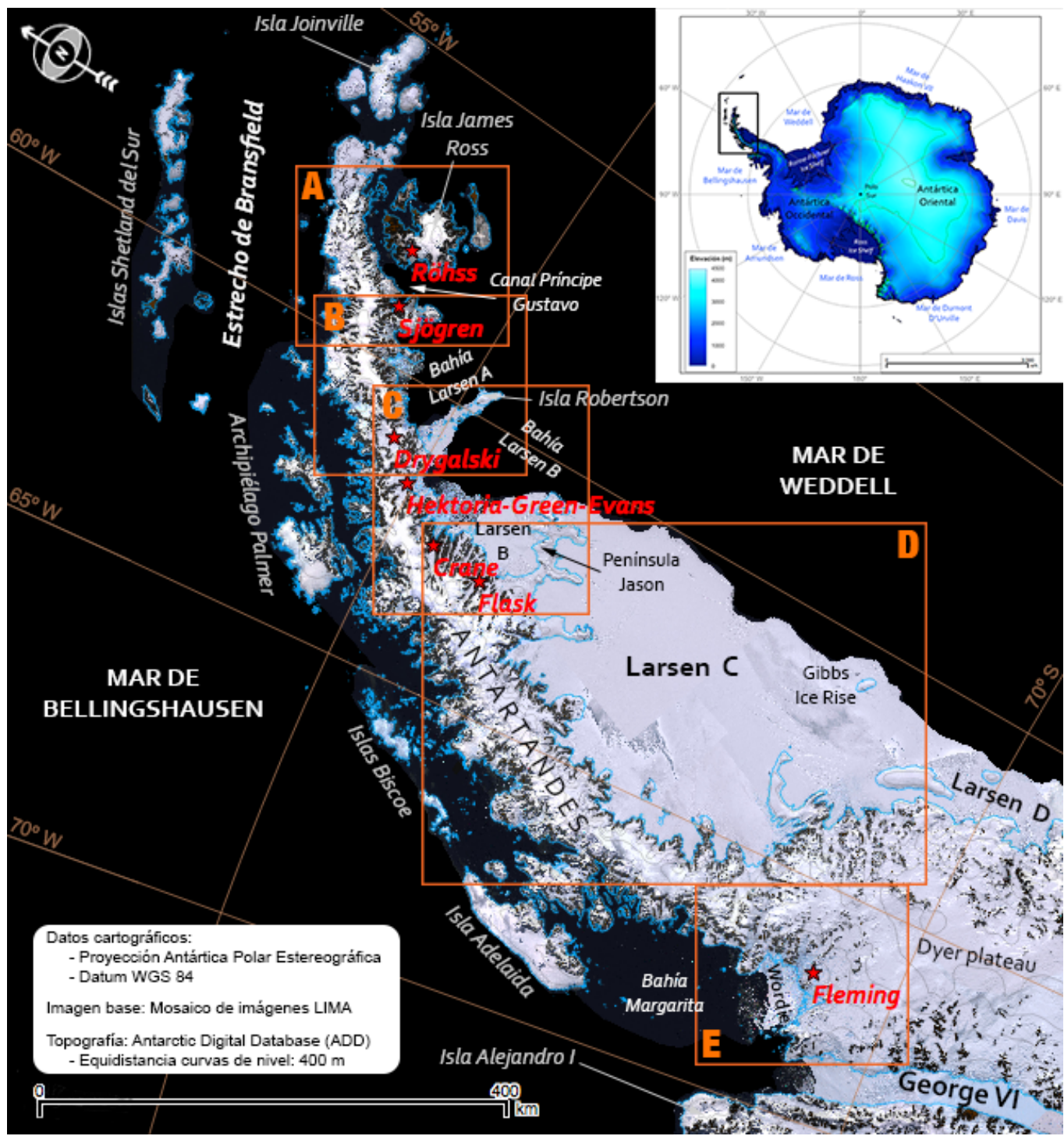

Fig. 1. Mapa del sector norte de la Península (al norte de los $70^{\circ} \mathrm{S}$ ) con las ubicación de las principales plataformas de hielo, las desintegradas y las que aún perduran, y sus glaciares tributarios (en color rojo). En naranja se señalan las cinco zonas cartografiadas (A-E) en este trabajo.

plateau de Siberia al noreste de Asia. Por otro lado, en el Hemisferio Sur, la Península Antártica y el Mar de Bellingshausen (Vaughan et al. 2003). Para este último caso, cabe resaltar el fuerte gradiente climático que existe entre la costa oeste y la costa este, ya que el mencionado rápido calentamiento regional no afecta por igual en ambas partes, siendo la costa occidental y el mar aledaño de Bellingshausen donde es posible observar la clara tendencia del calentamiento climático regional (Morris \& Vaughan, 2003).
Los datos meteorológicos de la estaciones científicas de la costa oeste de la Península muestran un calentamiento de la superficie cercano a los $3^{\circ} \mathrm{C}$ desde 1950, que es además sustancialmente más rápido que ninguna otra región en la Antártica (Vaughan et al. 2001), y probablemente más sostenido que en Groenlandia o Alaska (Knight, 2006). Asimismo, la costa oeste de la PA, al contrario que la oriental, muestra un calentamiento máximo en el invierno austral, que ha sido atribuido a la reducción de la concentración del 
hielo marino en el Mar de Bellingshausen, ya que la zona de mayor tendencia negativa en la duración del hielo marino coincide con las dos estaciones (Faraday y Rothera) que muestran la mayor tendencia de calentamiento (Vaughan et al. 2003). Más aún, como muestran los datos hidrográficos recolectados por Meredith y King (2005), los cambios observados en el Mar de Bellingshausen, tienen una profunda coincidencia con el aumento de la temperatura atmosférica, provocando según los autores una retroalimentación positiva al calentamiento climático actual, que no sólo se restringe a la atmósfera.

Teniendo en cuenta la conexión establecida entre la temperatura del aire y la distribución del hielo marino, y la singularidad de los últimos 50 años en los 1.800 años previos, Vaughan y colaboradores (2003) proponen los siguientes mecanismos de refuerzo para explicar el presente calentamiento: 1) Cambio de la circulación oceanográfica, con un flujo más templado del Agua Profunda Circumpolar hacia las plataformas continentales, que podría influir en la cubierta del hielo marino en la costa oeste; 2) Cambio de la circulación atmosférica, mostrando un significante incremento de los vientos del oeste y mayores condiciones ciclónicas, unido al incremento de la frecuencia de las precipitaciones en invierno y el fuerte vínculo con el estado de ENSO (El Niño Southern Oscillation); 3) Feedback aire - hielo marino, ya que la ausencia de este último permite la transferencia de calor del océano a la atmósfera durante el invierno y disminuye el albedo regional, generando una retroalimentación positiva hacia el calentamiento, con temperaturas del aire más altas y menor producción de hielo marino (Vaughan et al. 2003).

En este contexto, la sensibilidad de la PA se hace visible en los cambios recientes de la criosfera. Un estudio completo elaborado por Cook y colaboradores (2005), da a conocer que el $87 \%$ de los glaciares (del tipo tidewater, es decir con sus frentes en contacto directo con el mar) estudiados en la Península, ha retrocedido durante los últimos 50 años, y que además, el promedio de las tasas de retroceso ha acelerado (Pritchard \& Vaughan, 2007). Más, teniendo en cuenta que las tasa de fusión de estos glaciares depende críticamente de la temperatura del océano con el que están en contacto (Shepherd et al. 2004). Este retroceso de los glaciares está de acuerdo con la reducción de la cubierta de nieve permanente y la extensión de la estación estival, que genera mayor cantidad de aguas de fusión y lubrica el basamento de los glaciares aumentando su velocidad de flujo (Rott et al. 2002; De Angelis \& Skvarca, 2003). Es más, en décadas recientes, siete de las doce plataformas que rodean la PA han retrocedido significantemente o se han desintegrado totalmente, y algunos de estos colapsos se han tomado como hechos inusuales, dentro del contexto del Holoceno (últimos 11.700 años antes del presente), siendo atribuido su pérdida a los cambios atmosféricos y oceánicos recientes (Cook \& Vaughan, 2010).

\section{CASO ESTUDIADOS}

Las variaciones recientes de las plataformas de hielo en la PA han sido recopilados por varios autores en diversas publicaciones (Vaughan \& Doake, 1996; Ferrigno et al. 2005; Cook \& Vaughan, 2010; Depoorter et al. 2013). Es por ello que en el presente trabajo se han estudiado estas variaciones de las principales plataformas de la Península (al norte de los $70^{\circ} \mathrm{S}$ ), y después, se ha complementado con las investigaciones existentes de la evolución que tuvieron los glaciares tributarios que alimentaban dichas plataformas (Fig. 1).

Las plataformas estudiadas en la costa este son, de norte a sur, las ya desintegradas Príncipe Gustavo y Larsen A en 1995 (Glasser et al. 2011; Vaughan \& Doake, 1996), la relicta Larsen B después del gran colapso sufrido en 2002 (Rignot et al. 2004), y la más meridional Larsen C que aún parece presentar una dinámica estable (Cook \& Vaughan, 2010). Por otro lado, en la costa oeste, se ha tomado en cuenta la plataforma de hielo Wordie que en 1992 perdió gran parte de su amplia extensión (Ferrigno et al. 2008). Se ha decidido omitir las plataformas de hielo más septentrionales de Müller y Jones por su menor extensión $\left(<100 \mathrm{~km}^{2}\right)$.

A su vez, los glaciares tributarios elegidos para complementar este estudio son los siguientes: Röhss y Sjögren para la plataforma Príncipe Gustavo (Cook et al. 2005; Glasser et al. 2011), Drygalski para Larsen A (Rott et al. 1996; De Angelis \& Skvarca, 2003), la confluencia de los glaciares Hektoria- 
Tabla 1. Cambios registrados en las áreas de las principales plataformas de hielo de la Península por décadas (adaptado de Alison J. Cook, British Antarctic Survey, 2010).

\begin{tabular}{|c|c|c|c|c|c|c|c|c|c|}
\hline \multirow{2}{*}{$\begin{array}{l}\text { Plataforma de } \\
\text { hielo }\end{array}$} & \multicolumn{7}{|c|}{ Área en $\mathrm{km}^{2}$ (por décadas) } & \multirow{2}{*}{$\begin{array}{c}\text { Cambio Total } \\
\left(\mathrm{km}^{2}\right)\end{array}$} & \multirow{2}{*}{$\begin{array}{c}\% \\
\text { restante }\end{array}$} \\
\hline & $1950 \mathrm{~s}$ & 1960s & $1970 s$ & $1980 \mathrm{~s}$ & $1990 \mathrm{~s}$ & $2000 s$ & $2008 / 2009$ & & \\
\hline Príncipe Gustavo & 1.632 & 1.299 & 1.328 & 1.019 & 665 & 276 & 11 & -1.621 & 1 \\
\hline Larsen A & 4.021 & 3.736 & 3.873 & 3.394 & 926 & 638 & 397 & -3.624 & 10 \\
\hline Larsen B & & 11.573 & 11.958 & 12.190 & 8.299 & 4.429 & 2.407 & -9.166 & 21 \\
\hline Larsen C & & 56.131 & 58.036 & 50.241 & 51.246 & 51.593 & 50.837 & -5.295 & 91 \\
\hline Wordie & 1.420 & 1.917 & 1.538 & 827 & 906 & 312 & 139 & -1.281 & 10 \\
\hline Área Total & 74.777 & 74.656 & 76.733 & 67.671 & 62.042 & 57.248 & 53.791 & & \\
\hline Cambio Total & & -121 & 2.077 & -9.062 & -5.629 & -4.794 & -3.457 & -20.987 & 71,9 \\
\hline
\end{tabular}

Green-Evans, el glaciar Crane y Flask para Larsen B (Rignot et al. 2004; Scambos et al. 2004), y el glaciar Fleming para la plataforma Wordie (Doake \& Vaughan, 1991; Rignot et al. 2005).

\section{1) DATOS Y MÉTODOS}

Como se ha citado, muchas plataformas de hielo han sido monitoreadas y documentadas individualmente en diversas publicaciones, pero hasta hace poco no había ninguna recopilación consistente de estos cambios medidos para todas las plataformas de la PA, y en un período de tiempo regular. Así, en el año 2010, Cook y Vaughan publicaron la compilación de los datos obtenidos de las mediciones de las plataformas de hielo, a partir del set de datos del cambio costero recabado por el USGS (United States Geological Survey) y actualizado con la utilización de imágenes Envisat ASAR del período 2008/2009.

Estos datos se pueden obtener en la Base de Datos Digital (ADD: Antarctic Digital Database) del SCAR (Scientific Comittee on Antarctic Research), consiguiendo así recopilar la información necesaria para este estudio (Tabla 1).

Además de los datos mencionados, el método utilizado en este estudio ha sido la elaboración de mapas temáticos para visualizar el frente de las plataformas de hielo, con el programa Quantum GIS (versión 1.8). Para la imagen base se ha obtenido el Mosaico de Imágenes LIMA (Landsat Image Mosaic of Antarctica), es decir, un mosaico de la Antártica elaborado con imágenes Landsat 7 ETM+ (bandas 1, 2 y 3) del período 1999-2003.
Asimismo, las curvas de nivel, que muestran una equidistancia de 400 metros, han sido obtenidas de la cartografía topográfica del ADD, y las denominaciones toponímicas se complementaron con informaciones obtenidas de los mapas del BAS (British Antarctic Survey) y la base de datos del SCAR (Composite Gazetteer of Antarctica).

\section{2) ANÁLISIS}

El siguiente análisis se lleva a cabo de forma geográfica, siguiendo un orden latitudinal de norte a sur. Así, comenzando por la plataforma más septentrional del Canal Príncipe Gustavo, se sigue con Larsen A y B, hasta Larsen C en la costa oriental, y después saltar al margen occidental, donde se encuentra la relicta plataforma de hielo Wordie, cerca de los $70^{\circ} \mathrm{S}$.

Cabe resaltar, a primera vista, el marcado retroceso que sufrieron las plataformas más septentrionales de la costa este y la plataforma Wordie en al costa occidental. Además de observar una tendencia negativa para la plataforma de hielo Larsen B, si bien es cierto que la plataforma Larsen $\mathrm{C}$ presenta aún signos de estabilidad (Fig. 2).

\section{1) PLATAFORMA DE HIELO PRÍNCIPE GUSTAVO}

La plataforma de hielo Príncipe Gustavo (64²2' S, 58³0' O) era la plataforma más septentrional y la primera que mostró signos de retroceso. Aunque en 1945 tenía $2.100 \mathrm{~km}^{2}$ y era la continuación de la más amplia plataforma 


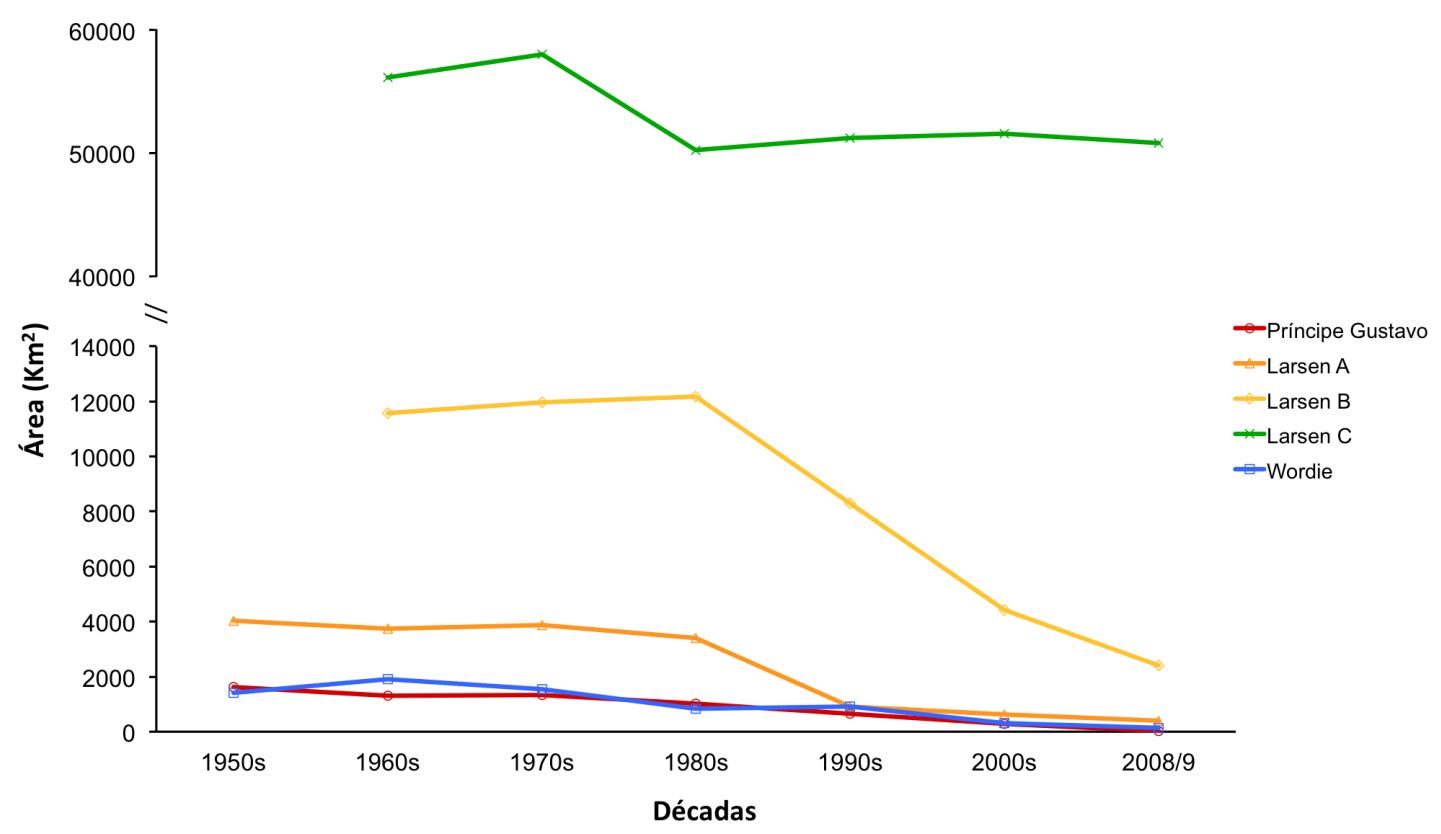

Fig. 2. Variaciones recientes de las plataformas de hielo.

Larsen A, retrocedió constantemente desde mediados del siglo XX (Cooper, 1997; Cook \& Vaughan, 2010). En 1995, finalmente colapsó, permitiendo que el agua libre de hielo ocupase el canal del mismo nombre entre la isla James Ross y la Península Antártica (Glasser et al. 2011). En 2008 , solamente quedaban $11 \mathrm{~km}^{2}$ de la plataforma relicta, confinada en nichos aislados (Fig. 3-A).

El retroceso fue rápido desde 1945 a 1961, seguido de un período de unos 10-15 años donde incluso aumentó su superficie, como es observable en el resto de las plataformas de hielo. Este breve periodo fue seguido de un constante retroceso hasta su mencionado colapso en 1995.

Quizás la diferente morfología de los márgenes norte y sur también pueda explicar este proceso; en el norte, los márgenes del hielo estaban fijados en el estrecho tramo entre el cabo Obelisk y la costa opuesta, mientras que en el sur, los márgenes terrestres son más abiertos.

Al mismo tiempo, después del colapso, los glaciares tributarios que nutrían la plataforma presentaron un aumento significativo en la velocidad de flujo del hielo y un relacionado adelgazamiento de sus espesores. Es así el ejemplo de los glaciares Sjögren (en la PA) y Röhss (en la isla James Ross).

El glaciar Sjögren (64¹4' S, 5854' O), que hasta 1993 fluía a la plataforma Príncipe Gustavo, paso a ser un glaciar flotante (tidewater glacier) retrocediendo más de $13 \mathrm{~km}$ en los años siguientes (Cook et al. 2005). De Angelis y Skvarca (2003) midieron el desplazamiento de grietas (crevasses) de la superficie del hielo entre Febrero del 2000 y Septiembre del 2001, obteniendo velocidades de 1,8-2,4 m/día.

Por otro lado, el glaciar Röhss (64º8' S, $58^{\circ} \mathrm{O}$ ), después del colapso de la plataforma, retrocedió rápidamente, adelgazó y aceleró su dinámica debido a la extracción del estrés basal dado por la plataforma de hielo y por el incremento de pérdida de masa por la transición a un glaciar del tipo tidewater, con desprendimiento de témpanos o calving (Glasser et al. 2011). Estos mismos autores, midieron el retroceso del frente glaciar entre Enero de 2001 y Marzo de 2009, estimado en unos $15 \mathrm{~km}$, junto a una pérdida de más del $70 \%$ de su área.

\section{2) PLATAFORMA DE HIELO LARSEN A}

La plataforma de hielo Larsen A (64\% $40^{\prime}$ S, $60^{\circ} \mathrm{O}$ ), aledaña a Príncipe Gustavo, se extendía desde el Cabo Longing hasta la isla Robertson donde se unía con Larsen B en Seal Nunataks. Permaneció relativamente estable hasta la década de 1980, cuando comenzó a retroceder por 
etapas, y desintegrándose casi en su totalidad con un gran colapso entre Enero y Febrero de 1995. En este período más de $2.000 \mathrm{~km}^{2}$ de hielo fueron a la deriva en pocas semanas (Cook \& Vaughan, 2010).

La parte de la plataforma de hielo dentro de la Ensenada Larsen (Larsen Inlet) también continuó retrocediendo después de desconectarse

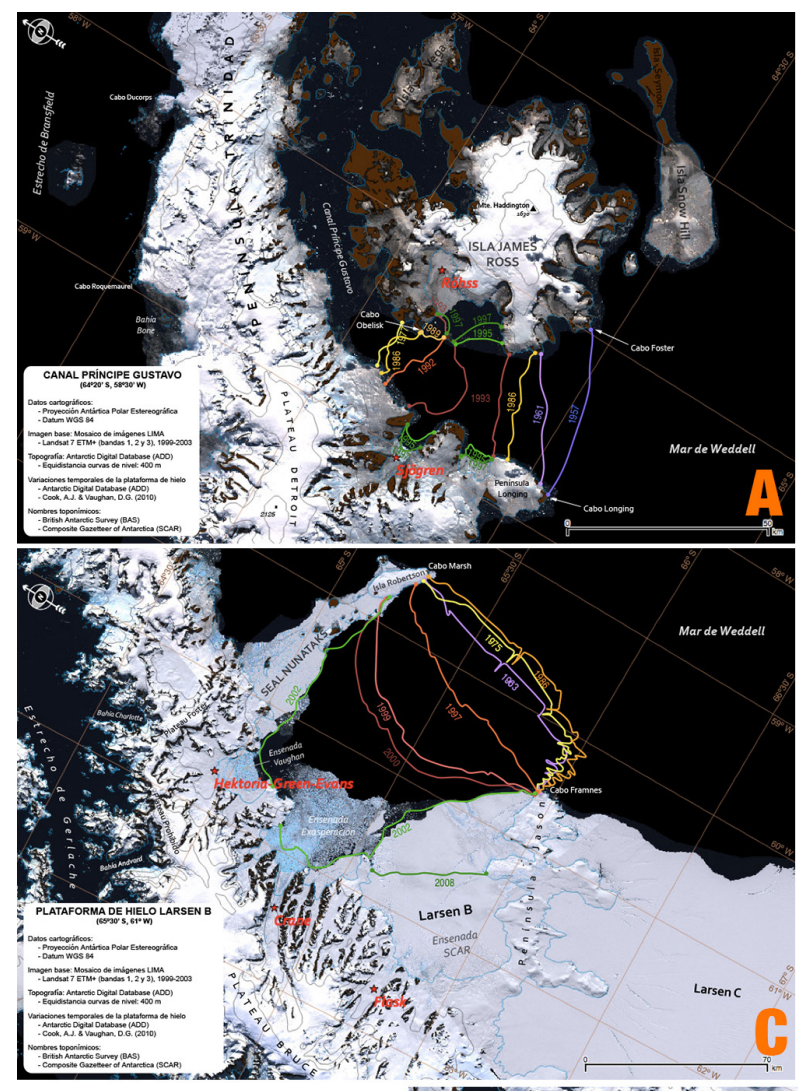

del resto de la plataforma Larsen A, cuando sufrió el mayor retroceso entre 1987 y 1989 (Fig. 3-B).

Después del colapso de 1995, la velocidad de retroceso disminuyó debido a que los restos relictos de la plataforma se encontraban fijados a puntos de sujeción (pinning points). Es el caso del área conocida como Seal Nunataks donde la plataforma aún perdura, si bien no es totalmente conocido si el

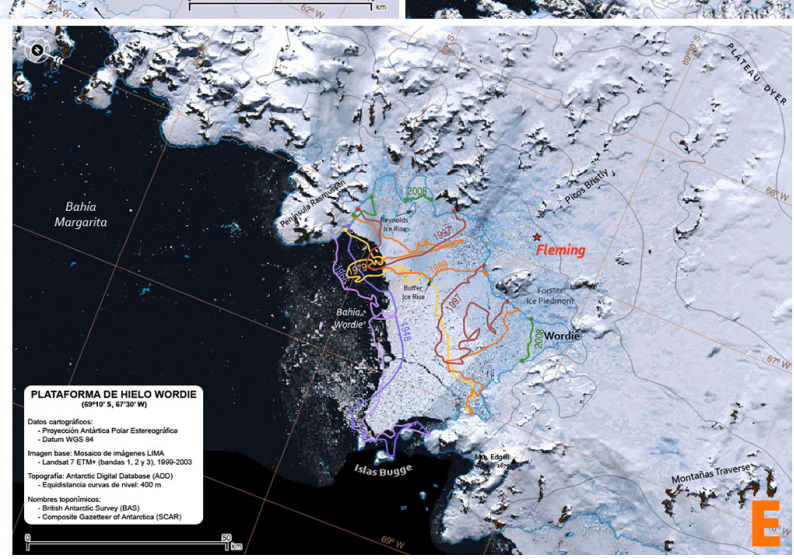

Fig. 3. Variaciones de las plataformas de hielo por cada zona cartografiada; A) Canal Príncipe Gustavo, B) Larsen A, C) Larsen B, D) Larsen C, y E) Wordie. 
hielo flota o no (Cook \& Vaughan, 2010).

Asimismo, el glaciar Drygalski (64\% $43^{\prime} \mathrm{S}$, $60^{\circ} 44^{\prime}$ O), una vez que la plataforma colapsó, sufrió un aceleramiento del flujo y adelgazamiento del espesor, con una pérdida de $24 \mathrm{~km}^{2}$ de superficie y coincidiendo con más del triple de incremento en la velocidad del hielo entre 1995 y 1999. Es por ello que en las fotografías tomadas por De Angelis y Skvarca en 2002 es posible observar terrazas de hielo colgadas, dando a conocer hasta que altura llegaba el espesor del hielo unos años atrás (De Angelis \& Skvarca, 2003).

Este hecho también fue observado por los mismos autores para el caso del glaciar Sjögren, siendo evidencia de glaciares de surgimiento (surge glaciers).

\section{3) PLATAFORMAS DE HIELO LARSEN B}

La plataforma de hielo Larsen B (653' S, $\left.61^{\circ} \mathrm{O}\right)$, se encontraba al sur de Larsen A, desde la isla Robertson (y Seal Nunataks) hasta la península Jason, donde a día de hoy se encuentra confinada en la Ensenada SCAR (SCAR Inlet) y favorecido por la morfología en forma de herradura que encierra en parte a la plataforma.

Hasta 1992, la plataforma se mantuvo relativamente estable ya que, al contrario que Larsen A, Larsen B avanzó incluso, con una superficie aproximada de $12.000 \mathrm{~km}^{2}$. Sin embargo, en 1995 se desprendió un iceberg tabular de unos $1.700 \mathrm{~km}^{2}$ que fue seguido de un patrón de retroceso parecido al observado para Larsen A, culminando con un dramático colapso de aproximadamente $3.250 \mathrm{~km}^{2}$ en poco días en Febrero de 2002 (Fig. 3-C).

Es a partir de este colapso cuando los glaciares adyacentes que nutrían la plataforma presentan un significativo aceleramiento en sus flujos y adelgazamiento respectivo, tal y como se ha visto en los casos anteriores.

Es el caso de los glaciares hoy en día faltos

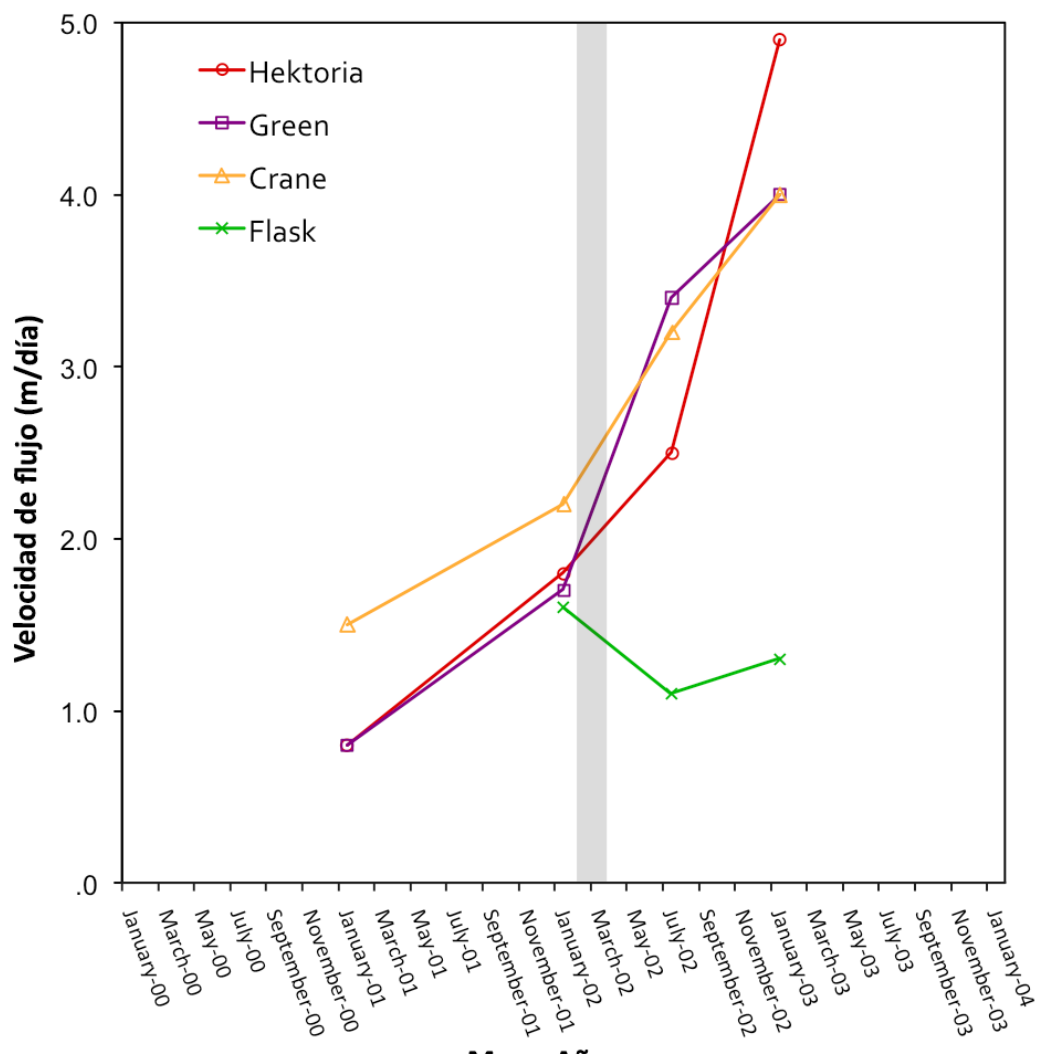

Mes y Año

Fig. 4. Velocidad de flujo de los glaciares tributarios a la plataforma de hielo Larsen B. La columna gris indica la fecha del colapso (modificado de Scambos et al. 2004). 
del mencionado "efecto tapón" (contención de la plataforma en la dinámica de los glaciares tributarios), como son la cuenca compuesta por los glaciares Hektoria-Green-Evans y el glaciar Crane, mientras que el glaciar Flask, que aún fluye a la plataforma relicta en la Ensenada SCAR, no presenta mayor cambio. En el siguiente gráfico (Fig. 4) se puede observar el aceleramiento acentuado que sufrieron los glaciares mencionados, excepto el glaciar Flask, después del colapso de la plataforma Larsen B (Scambos et al. 2004).

Al respecto, Rignot y colaboradores (2004) mencionan que la aceleración generalizada de los glaciares coincide con la eliminación de las plataformas de hielo, mientras que los glaciares que aún se encuentran contenidos por ellos no han acelerado.

Según su estudio, los glaciares HektoriaGreen-Evans aceleraron 8 veces entre 2000 y comienzos de 2003, si bien después disminuyó esa tasa. El glaciar Crane duplicó su velocidad a comienzos de 2003 y triplicó a finales de 2003. En contraste, el glaciar Flask y el cercano Leppard, que todavía se encuentran contenidos por la parte remanente de la plataforma Larsen $\mathrm{B}$, no aceleraron entre 1996 y comienzos de 2003, si bien a finales de ese mismo año tanto estos glaciares como la plataforma aceleraron un $15 \%$, lo cual podría ser una señal de una posible transición hacia un flujo más rápido (Rignot et al. 2004).

\section{4) PLATAFORMAS DE HIELO LARSEN C}

La plataforma Larsen C (67³0' S, 62 $30^{\circ}$ O) es la plataforma de hielo más extensa en la PA, con más de $50.500 \mathrm{~km}^{2}$. Se extiende desde la península Jason en el norte hasta el Gibbs Ice Rise al sur, donde se fusiona con la plataforma Larsen D.

Hasta la fecha, no muestra una evidencia clara de retroceso determinado por el clima, ya que las variaciones en el frente del hielo siguen un patrón normal dentro de las variaciones de las plataformas de hielo (Fig. 3-D). El mayor cambio ocurrió en 1986 cuando se desprendieron dos icebergs gigantes; el más grande tenía un área aproximada de $6.520 \mathrm{~km}^{2}$ y el otro unos 1.260 $\mathrm{km}^{2}$ (Skvarca, 1994). Después de este evento no ha habido más desprendimientos (calving) de tales magnitudes, es más, el frente del hielo ha avanzado lentamente. Actualmente contiene un $90 \%$ de su tamaño original (en 1963) y parece que permanece estable (Cook \& Vaughan, 2010).

Skvarca (1994), al observar que no hay ningún glaciar tributario significante que fluya a la plataforma desde la península Jason, argumenta que la plataforma de hielo se sustenta por la acumulación de nieve que cae sobre ella. Actualmente, se ha podido observar que la plataforma se encuentra bastante fisurada y agrietada, fuertemente influenciada por la entrada de hielo terrestre, principalmente desde las ensenadas Cabinet, Mill, Whirlwind y Mobiloil (Cook \& Vaughan, 2010).

Si bien se ha comentado que esta plataforma no presenta grandes cambios a primera vista, algunos estudios muestran que la plataforma pueda comenzar a retroceder en años venideros. Es el caso del estudio de Shepherd y colaboradores (2003), que si bien establecen que las tasas de fusión basal son inciertas, el amplio derretimiento provocado por el océano ha adelgazado la plataforma (y lo seguirá haciendo). Según sus predicciones, si la fusión basal continúa en la tasa estimada de 0,78 m/año, Larsen $C$ se acercará al espesor del momento del colapso de Larsen B en unos 100 años, o más rápidamente con un océano más cálido (Shepherd et al. 2003).

\section{5) PLATAFORMA DE HIELO WORDIE}

La plataforma de Wordie (69¹0' S, 67³0' O), fue la plataforma de hielo septentrional más extensa $\left(>100 \mathrm{~km}^{2}\right)$ en la costa occidental de la PA, desintegrado en una serie de eventos durante las décadas de 1970 y 1980, y desde 1992 sólo quedaban algunos pedazos de plataforma remanentes desconectados entre sí (Cook \& Vaughan, 2010).

Este caso ha sido el retroceso más dramático observado entre todas las plataformas de hielo de la PA (Fig. 3-E), y ya en 1940, Fleming interpretó que la plataforma era un rasgo relicto por sus pobres condiciones de aspecto y sugirió que no sobreviviría por largo tiempo (Fleming, 1940).

La plataforma Wordie estaba alimentada por unos cuantos glaciares tributarios que se unían en tres unidades de entrada principales. En 1993, 
Vaughan concluyó que no había cambios en la dominancia relativa de los glaciares tributarios siguiendo al colapso de la plataforma de hielo $y$, por eso, que la plataforma no proveía una restricción significativa para estos glaciares (Vaughan, 1993).

Sin embargo, los estudios más recientes llevados a cabo por Rignot y colaboradores (2005) en uno de los glaciares tributarios, como es el caso del glaciar Fleming (69²5' S, 6640' O), han observado que la desintegración de la plataforma de hielo Wordie ha tenido sus consecuencias en el flujo de la parte alta (upstream) del glaciar. Para el año de estudio, el glaciar fluía 50\% más rápido que en el año 1974, antes del colapso principal de la plataforma. Por lo tanto, concluyen que los glaciares aceleraron siguiendo la desintegración de la plataforma de hielo y han estado adelgazando y perdiendo masa al océano en las últimas décadas, siendo un aporte fundamental que no debe ser omitido en los estudios de impacto sobre el nivel del mar (Rignot et al. 2005).

\section{DISCUSIÓN}

Una de las mayores discusiones que existe en las publicaciones que comprenden estudios de la conexión de las plataformas de hielo con la dinámica de los glaciares tributarios es la pregunta de cuál es el principal mecanismo que genera el retroceso y desintegración de las plataformas, generando la aceleración y adelgazamiento de los glaciares adyacentes.

A raíz de esta pregunta, muchos son los autores que han discutido diversas ideas. A día de hoy, seguramente, la más extendida y aceptada sea la propuesta por Vaughan \& Doake (1996), argumentando que el límite geográfico de las plataformas de hielo alrededor de la PA es debido a condiciones termales. Según los autores, y mejorado en un mapa por Morris \& Vaughan (2003), el presente límite de las plataformas de la Península sigue de cerca la isoterma $-9{ }^{\circ} \mathrm{C}$, además de observar que las plataformas que han retrocedido en los últimos 100 años están confinadas entre las isotermas $-5{ }^{\circ} \mathrm{C}$ y $-9{ }^{\circ} \mathrm{C}$, sugiriendo que el retroceso de las plataformas en la PA consiste en un calentamiento de $\sim 4{ }^{\circ} \mathrm{C}$ (Vaughan \& Doake, 1996). Es decir, las plataformas de hielo para que puedan mantener una dinámica estable deben estar cerca o por debajo del isoterma $-9{ }^{\circ} \mathrm{C}$, como es el caso de la plataforma Larsen $\mathrm{C}$; mientras que las que se encuentran confinadas entre las isoterma -5 ${ }^{\circ} \mathrm{C}$ y $-9{ }^{\circ} \mathrm{C}$ presentan signos de retroceso (como es el caso de Larsen B) o ya han sido desintegradas (Príncipe Gustavo, Larsen A y Wordie); y más allá de la isoterma $-5{ }^{\circ} \mathrm{C}$, nunca se ha formado una plataforma de hielo en los últimos 1.800 años BP (Fig. 5).

Así pues, el retroceso continuado de las plataformas de la PA ha sido ampliamente atribuido al mencionado calentamiento atmosférico reciente. Este hecho está confirmado por el retroceso de los frentes glaciares de la PA en el último medio siglo, que también ha mostrado, al igual que en el caso de las plataformas, que el límite de la isoterma $-9^{\circ} \mathrm{C}$ está descendiendo hacia el sur, siendo así que plataformas y glaciares hasta ahora intactos comienzan a mostrar posibles síntomas de inestabilidad (Cook et al. 2005; Pritchard \& Vaughan, 2007; Cook \& Vaughan, 2010). Aún así, la rapidez de esta migración sugiere que esta no sea la única clave del retroceso de los glaciares y plataformas en la región.

El calentamiento de las aguas oceánicas también puede ser importante. Los mares próximos a la PA, se han calentado a fines del siglo $\mathrm{XX}$, si bien no se conoce bien como esto ha podido contribuir a la desintegración de las plataformas. El incremento de temperaturas oceánicas debería incrementar las tasas de fusión basal y provocar que una plataforma adelgazase o retrocediese su línea de anclaje. Ambos procesos pueden incrementar los flujos extensivos, posibilitando el aumento de fracturas y eventos de calving (Cuffey \& Patterson, 2007).

Según Depoorter y colaboradores (2013), el derretimiento de las plataformas de hielo en la Antártica es provocado por la circulación de agua relativamente templada de tres diferentes formas: 1) La formación de hielo marino y la producción de una lámina de agua de alta salinidad (generado en el mismo proceso de formación de la banquisa), que llega a la línea de anclaje (grounding line) y pasa a formar parte del agua líquida de la plataforma, siendo ésta una mezcla entre las aguas de alta salinidad y las de fusión basal de la plataforma; 2) Incursión de la Corriente Circumpolar Profunda 


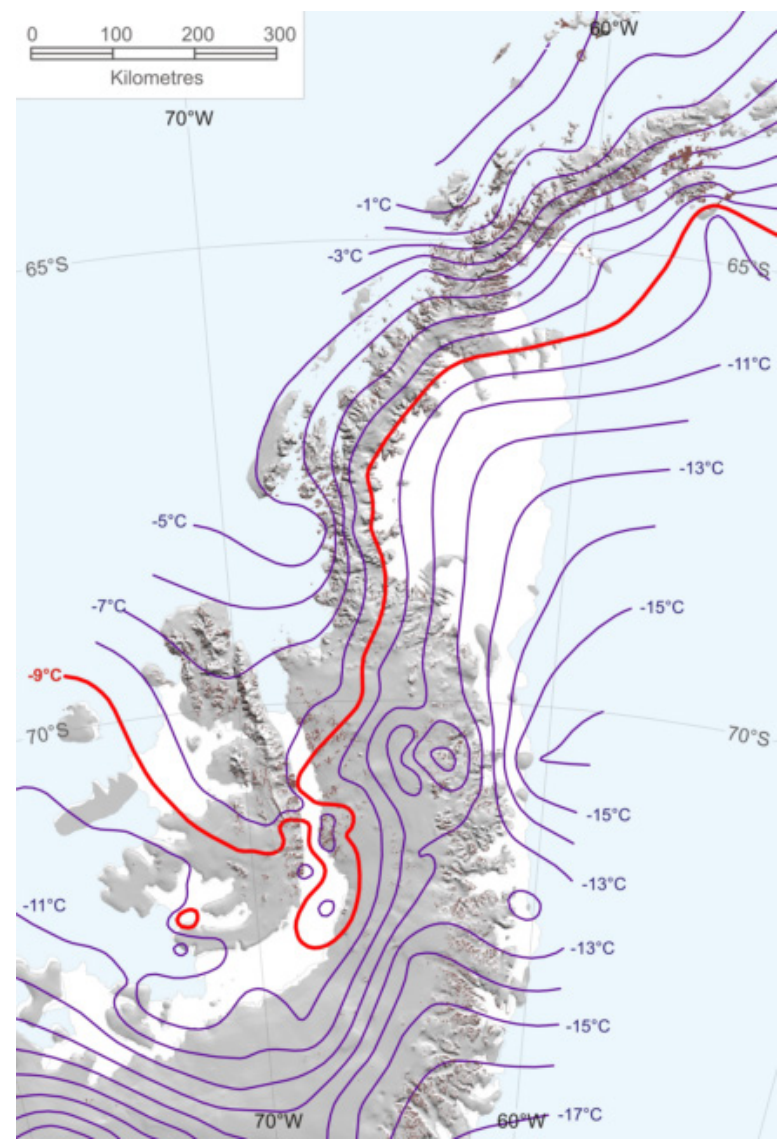

Fig. 5. Isotermas de la temperatura promedio anual en los últimos 2.000 años (recopilado por Morris y Vaughan, 2003; obtenido de Cook \& Vaughan, 2010).

(Circumpolar Deep Water) en las cavidades basales de la plataforma de hielo; 3) Mezcla inducida por el viento y la marea en las cercanías del borde de la plataforma. Si bien es cierto que entre la línea de anclaje y el borde de la plataforma, el agua basal es enfriado hasta el punto donde puede darse la formación de hielo recongelado.

Otro punto fundamental a tener en cuenta es la importancia que tienen los conocidos como pinning points (puntos de sujeción) que suponen los mencionados ice rises y rumples (como arrugas, menores en tamaño a los anteriores), ya que cuando una plataforma se desancla de estas protuberancias, además de reducir el cizallamiento provocado por las paredes laterales que lo contienen, la contención de la plataforma se reduce provocando una mayor inestabilidad y tendencia al colapso (Doake \& Vaughan, 1991).
Además, cabe resaltar las diferencias resultantes según la configuración de la bahía donde se establece la plataforma, ya que una bahía en forma de herradura y con algunas protuberancias para el anclaje permitirá más fácilmente contener un plataforma, al contrario que una bahía arqueada y con pocas opciones de sujeción (Cook \& Vaughan, 2010).

La textura de la superficie, el espesor del hielo y la velocidad del hielo también juegan un papel importante en la determinación del comportamiento del retroceso de una plataforma de hielo. Es posible también que el debilitamiento reológico de los márgenes cizallados pueda causar un incremento en las tasas de flujo del hielo (como ocurrió en la plataforma Larsen B), generando que el desarrollo de grietas y la ruptura de las fisuras entre unidades de flujo pueda generar las precondiciones para el colapso de una plataforma (Glasser \& Scambos, 2008).

Cuando esto ocurre, la desintegración de una plataforma de hielo supone eliminar el citado "efecto tapón" que éstas generan en los glaciares adyacentes, quedando en una situación de inestabilidad dinámica. Por ello, aceleran su flujo (con casos mencionados de más del doble de la velocidad) para extenderse, perdiendo así espesor rápidamente $\mathrm{y}$, por tanto, volumen, con un mayor aporte de hielo a las aguas del mar (Gudmundsson, 2013).

En este contexto de continuo retroceso de las plataformas y la importancia que tienen estas sobre los glaciares adyacentes, cabe resaltar el ejemplo del glaciar Pine Island, que si bien no es parte de la PA, es una de las zonas clave de la Antártica. El glaciar Pine Island $\left(75^{\circ} 10^{\prime} \mathrm{S}, 100^{\circ} \mathrm{O}\right)$ es la mayor corriente de hielo (ice stream) de la Antártica Occidental, siendo subrayado como vulnerable al cambio climático y posible desencadenador de la desintegración del Hielo Continental de la Antártica Occidental (WAIS en sus siglas en inglés). El flujo de hielo fluye rápidamente hacia la bahía Pine Island, en el Mar de Amundsen, encontrándose libre de cualquier plataforma de hielo en su unión con el océano y sobre el lecho subglacial bien por debajo del nivel del mar. Esta configuración del flujo es teóricamente inestable, porque el retroceso de su línea de anclaje puede ser irreversible (Rignot, 1998). 


\section{CONCLUSIONES}

El retroceso de las plataformas de hielo y el posterior aceleramiento y adelgazamiento de los glaciares es un tema en auge en los estudios glaciológicos del cambio climático que abarcan la Península Antártica.

Los datos sugieren que la respuesta de los glaciares tributarios a la desintegración de las plataformas de hielo siguen una escala temporal anual o decadal, y que estos son a su vez muy sensitivos a los colapsos, ya que los cambios recientes pueden ser parte de una conducta cíclica mayor (Cook et al. 2005). Además, las mediciones de velocidad del flujo de hielo hechas en los glaciares citados dan a conocer una aceleración de más del doble de su velocidad (siendo triplicado en muchos casos e incluso ocho veces más en casos concretos) y adelgazamiento del espesor en los meses siguientes al colapso, si bien en los meses anteriores también pueden observarse algunos síntomas de la posible desintegración que está por suceder.

Análogamente, el desarrollo de las plataformas de hielo de la PA en el Holoceno tardío parece que está relacionado con una escala milenaria de variabilidad climática en la región (Mulvaney et al. 2012). Los mismos autores resaltan que la rapidez del calentamiento en la PA es inusual pero no sin precedentes, ya que la historia climática del Holoceno presenta tasas de calentamiento similares o incluso mayores a las actuales.

La tasa de retroceso de las plataformas no parece que simplemente esté relacionado a la tasa de cambio de las condiciones climáticas, ya que otros procesos también toman parte en su configuración y comportamiento, es por ello que están sujetas a diferentes mecanismos. La idea más aceptada corresponde al límite de viabilidad termal de la temperatura del aire que parece ser el principal causante del retroceso de las plataformas, si bien explica y predice el patrón general de estos cambios (Cook \& Vaughan, 2010). Por tanto, es de principal interés profundizar en los mecanismos de refuerzo que retroalimentan positivamente al citado mecanismo principal, para entender el retroceso de las plataformas en la PA, y así poder mejorar los modelos y proyecciones de los cambios futuros que se puedan dar en las más extensas plataformas y glaciares flotantes del Hielo Continental de la Antártica Occidental (WAIS).

Así pues, conociendo que las proyecciones para la PA son de aumento de las temperaturas del aire y oceánicas, junto a la intensificación de los mecanismos de refuerzo, se concluye que el retroceso de las plataformas y su conexión con los glaciares tributarios continuará hacia el sur, siendo que plataformas que hasta el momento presentaban condiciones estables en los últimos 1.800 años BP, pueden comenzar a presentar signos de inestabilidad, como en el caso de la parte norte de Larsen C o las plataformas de hielo del Mar de Amundsen (Rignot, 1998; Cook \& Vaughan, 2010). Esto puede generar nuevos colapsos en años venideros, y que la velocidad de movimiento de esos glaciares que tributan aumente exponencialmente, generando un balance de masa negativo de los glaciares tributarios y que, en el caso de encontrarse sin el "efecto tapón" de una plataforma de hielo, suponga un aporte de agua a tener en cuenta en los modelos de incremento del nivel del mar (Rignot et al. 2004).

\section{AGRADECIMIENTOS}

Este ensayo es parte de la asignatura Geografía Antártica del Programa de Magíster en Ciencias Antárticas mención Glaciología de la Dirección de Programas Antárticos y Subantárticos (UMAG). Debo dar las gracias al profesor Ricardo Jaña por la motivación puesta en este trabajo, al profesor Gino Casassa por la rigurosa revisión, al compañero Erling Johnson por su paciencia y a mi amigo Diego Espinoza por las largas conversaciones mantenidas en torno a este tema.

\section{LITERATURA CITADA}

Cook, A. J., Fox, A. J., Vaughan, D. G., \& Ferrigno, J.G. (2005), Retreating glacier fronts on the Antarctic Peninsula over the past half-century. Science, 308, 541-544.

Cook, A. J., \& Vaughan, D. G. (2010). Overview of areal changes of the ice shelves on the Antarctic Peninsula over the past 50 years. The Cryosphere, 4, 77-98.

Cook, A. J., Murray, T., Luckman, A., Vaughan, 
D. G., \& Barrand, N. E. (2012). A new 100$\mathrm{m}$ Digital Elevation Model of the Antarctic Peninsula derived from ASTER Global DEM: methods and accuracy assessment. Earth System Science Data, 4(1), 129-142.

Cooper, A. P. R. (1997) Historical observations of Prince Gustav Ice Shelf. Polar Record, 33(187), 285-294.

Cuffey, K., \& Patterson, W. (2010). The Physics of Glaciers ( $4^{\mathrm{a}}$ edn.). Burlington, Estados Unidos: Editorial Elsevier B.V.

De Angelis, H., \& Skvarca, P. (2003). Glacier Surge after ice shelf collapse. Science, 299, 1560-1562.

Depoorter, M. A., Bamber, J. L., Griggs, J. A., Lenaerts, J. T. M., Ligtenberg, S. R. M., van den Broeke, M. R., \& Moholdt, G. (2013). Calving fluxes and basal melt rates of Antarctic ice shelves. Nature, 502, 89-92.

Doake, C. S. M., \& Vaughan, D. G. (1991). Rapid disintegration of the Wordie Ice Shelf in response to atmospheric warming. Nature, 350(6316), 328-330.

Dupont, T. K., \& Alley, R. B. (2005). Assessment of the importance of ice-shelf buttressing to ice-sheet flow. Geophysical Research Letters, 32, 4 pp.

Ferrigno, J. G., Williams, R. S. Jr., \& Fox, A. (2005). Coastal-change and glaciological maps of the Antarctic Peninsula. U.S. Geological Survey, Fact Sheet ,017-02, 2 pp.

Ferrigno, J. G., Cook, A. J., Foley, K. M., Williams, R. S. J., Swithinbank, C., Foley, K. M., Fox, A. J.,...Sievers, J. (2006). Coastalchange and glaciological map of the Trinity Peninsula area and South Shetland Islands, Antarctica: 1843-2001. U.S. Geological Survey, Geologic Investigations Map Series, Map I-2600-A, 32 pp.

Ferrigno, J. G., Cook, A. J., Mathie, A. M., Williams, R. S. J., Swithinbank, C., Fox, A. J., Thomson, J. W., \& Sievers, J. (2008). Coastal-change and glaciological map of the Larsen Ice Shelf area, Antarctica: 19402005. U.S. Geological Survey, Geologic Investigations Map Series, Map I-2600-B, 28 pp.

Fleming, W. L. S. (1940). Relic glacial forms on the western seaboard of Graham Land. Geographic Journal, XCVI, 93-101.

Glasser, N. F., \& Scambos, T. A. (2008). A structural glaciological analysis of the 2002 Larsen B ice-shelf collapse. Journals of Glaciology, 54, 3-16.

Glasser, N. F. Scambos, T. A., Bohlander, J., Truffer, M., Pettit, E., \& Davies, B. J. (2011). From ice-shelf tributary to tidewater glacier: continued rapid recession, acceleration and thinning of Röhss Glacier following the 1995 collapse of the Prince Gustav Ice Shelf, Antarctic Peninsula. Journal of Glaciology, 57(203), 397-406.

Gudmundsson, G. H. (2013). Ice-shelf buttressing and the stability of marine ice sheets. The Cryosphere, 7, 647-655.

Hansen, J., Ruedy, R., Sato, M., \& Lo, K. (2010). Global Surface Temperature Change. Reviews of Geophysics, 48, 1-29.

Houghton, J. T., Ding, Y., Griggs, D. J., Noguer, M., van den Linden, P. J., Dai, X., Maskell, K., \& Johnson, C. A. (2001). Climate Change 2001: The Scientific Basis. Cambridge, Reino Unido: Editorial Cambridge University Press.

IPCC, 5th Assessment Report (2013) Climate Change 2013: The Physical Science Basis. Estocolmo, Suecia: Editorial Intergovernmental Panel on Climate Change.

Knight, P. G. (2006). Glacier Science and Environmental Change. Londres, Reino Unido: Editorial Blackwell Science Ltd.

Meredith, M. P., \& King, J. C. (2005). Rapid climate change in the ocean west of the Antarctic Peninsula during the second half of the 20th century. Geophysical Research Letters, 32, $5 \mathrm{pp}$.

Mann, M. E., Bradley, R. S., \& Hughes, M. K. (1999). Northern Hemisphere Temperatures during the Past Millenium: Inferences, Uncertainties and Limitations. Geophysical Research Letters, 26, 759-762.

Morris, E. M., \& Vaughan, D. G. (2003). Spatial and temporal variation of Surface Temperature on the Antarctic Peninsula and the limit of viability of ice shelves. Antarctic Research Series, 79, 61-68. 
Mulvaney, R., Abram, N. J., Hindmarsh, R. C. A., Arrowsmith, C., Fleet, L., Triest, J., Sime, L. C.,...Food, S. (2012). Recent Antarctic Peninsula warming relative to Holocene climate and ice-shelf history. Nature, 489, 141-144.

Pritchard, H. D., \& Vaughan, D. G. (2007). Widespread acceleration of tidewater glaciers on the Antarctic Peninsula. Journal of Geophysical Research, 112, 10 pp.

Rack, W., \& Rott, H. (2004). Pattern of retreat and disintegration of Larsen B ice shelf, Antarctic Peninsula. Annals of Glaciology, 39, 505510.

Riffenburgh, B. (2007). Encyclopedia of the Antarctic, Vol. 1 (A-K). Nueva York, Estados Unidos: Editorial Taylor \& Francis Group.

Rignot, E. J. (1998). Fast recession of a West Antarctic glaciar. Science, 281, 549-551.

Rignot, E., Casassa, G., Gogineni, P., Krabill, W., Rivera, A., \& Thomas, R. (2004). Accelerated ice discharge from the Antarctic Peninsula following the collapse of Larsen B ice shelf. Geophysical Research Letters, 31, 4 pp.

Rignot, E., Casassa, G., Gogineni, S., Kanagaratnam, P., Krabill, W., Pritchard, H., Rivera, A., Thomas, R., Turner, J., \& Vaughan, D. (2005). Recent ice loss from the Fleming and other glaciers, Wordie Bay, West Antarctic Peninsula. Geophysical Research Letters, 32, 4.

Rott, H., Skvarca, P., \& Nagler, T. (1996). Rapid collapse of Northern Larsen Ice Shelf, Antarctica. Sience, 271, 788-792.
Scambos, T. A., Bohlander, J. A, Shuman, C. A., \& Skvarca, P. (2004). Glacier acceleration and thinning after ice shelf collapse in the Larsen B embayment, Antarctica. Geophysical Research Letters, 31, 4.

Scambos, T. A., Haran, T. M., Fahnestock, M. A., Painter, T. H., \& Bohlander, J. A. (2007). MODIS-based Mosaic of Antarctica (MOA) data sets: Continent-wide surface morphology and snow grain size. Remote Sensing of Environment, 111(2-3), 242257.

Shepherd, A., Wingham, D., Payne, T., \& Skvarca, P. (2003). Larsen ice shelf has progressively thinned. Science, 302, 856-859.

Shepherd, A., Wingham, D., \& Rignot, E. (2004). Warm ocean is eroding West Antarctic Ice Sheet. Geophysical Research Letters, 31(23), 4.

Skvarca, P. (1994). Change and surface features of the Larsen Ice Shelf, Antarctica, derived from Landsat and Kosmos mosaics. Annals of Glaciology, 20, 6-12.

Vaughan, D. G. (1993). Implications of the breakup of Wordie Ice Shelf, Antarctica, for sea level. Antarctic Sciences, 5(4), 403-408.

Vaughan, D. G., \& Doake, C. S. M. (1996). Recent atmospheric warming and retreat of ice shelves on the Antarctic Peninsula. Nature, 379, 328-331.

Vaughan, D. G., Marshall, G. J., Connolley, W. M., Parkinson, C., Mulvaney, R., Hodgson, D. A., King, J. C.,...Turner, J. (2003). Recent Rapid Regional Climate Warming on the Antarctic Peninsula. Climate Change, 60, 243-274. 